Supplement of

\title{
Fluorescent bioaerosol particle, molecular tracer, and fungal spore concentrations during dry and rainy periods in a semi-arid forest
}

Marie Ila Gosselin et al.

Correspondence to: J. Alex Huffman (alex.huffman@du.edu)

The copyright of individual parts of the supplement might differ from the CC-BY 3.0 licence. 


\begin{tabular}{|c|c|c|c|c|c|c|c|c|}
\hline $\begin{array}{l}\text { Sample } \\
\text { Name }\end{array}$ & Start Time & End Time & $\begin{array}{l}\text { Temp } \\
\left({ }^{\circ} \mathrm{C}\right)\end{array}$ & $\begin{array}{l}\text { Relative } \\
\text { Humidity } \\
(\%)\end{array}$ & $\begin{array}{l}\text { Rain Amount } \\
\text { (Normalized) }\end{array}$ & $\begin{array}{l}\text { Leaf } \\
\text { Wetness } \\
(\mathrm{mV})\end{array}$ & $\begin{array}{l}\text { FAP } \\
\text { Number } \\
\text { Ratio } \\
\left(\mathrm{N}_{\mathrm{f}} / \mathrm{N}_{\text {tot }}\right)\end{array}$ & Category \\
\hline HiVol 1* & $\begin{array}{l}7 / 24 / 2011 \\
18: 53\end{array}$ & $\begin{array}{l}7 / 25 / 2011 \\
18: 00\end{array}$ & 17.8 & 50.1 & 0.002 & 283.04 & 0.058 & Dry \\
\hline HiVol 2 & $\begin{array}{l}7 / 25 / 2011 \\
18: 07\end{array}$ & $\begin{array}{l}7 / 26 / 2011 \\
18: 01\end{array}$ & 18.8 & 55.3 & 0.015 & 268.49 & 0.071 & Dry \\
\hline HiVol 3* & $\begin{array}{l}7 / 26 / 2011 \\
18: 07\end{array}$ & $\begin{array}{l}7 / 27 / 2011 \\
18: 07\end{array}$ & 15.8 & 62.6 & 0.008 & 308.61 & 0.166 & Rainy \\
\hline HiVol 4 & $\begin{array}{l}7 / 27 / 2011 \\
20: 00\end{array}$ & $\begin{array}{l}7 / 28 / 2011 \\
20: 00\end{array}$ & 16.2 & 71.3 & 0.031 & 316.77 & 0.144 & Rainy \\
\hline HiVol 5 & $\begin{array}{l}7 / 28 / 2011 \\
20: 03\end{array}$ & $\begin{array}{l}7 / 29 / 2011 \\
20: 03\end{array}$ & 16.1 & 71.3 & 0.000 & 323.43 & 0.102 & Rainy \\
\hline HiVol 6 & $\begin{array}{l}7 / 30 / 2011 \\
9: 39\end{array}$ & $\begin{array}{l}7 / 31 / 2011 \\
7: 59\end{array}$ & 18.2 & 64.7 & 0.000 & 265.93 & 0.111 & Rainy \\
\hline HiVol 7* & $\begin{array}{l}7 / 31 / 2011 \\
8: 02\end{array}$ & $\begin{array}{l}7 / 31 / 2011 \\
11: 57\end{array}$ & 25.5 & 47.1 & 0.000 & 264.07 & 0.053 & Dry \\
\hline HiVol 8* & $\begin{array}{l}7 / 31 / 2011 \\
12: 01\end{array}$ & $\begin{array}{l}7 / 31 / 2011 \\
16: 05\end{array}$ & 28.5 & 60.2 & 0.000 & 261.54 & 0.037 & Dry \\
\hline HiVol 9 & $\begin{array}{l}7 / 31 / 2011 \\
16: 08\end{array}$ & $\begin{array}{l}7 / 31 / 2011 \\
20: 04\end{array}$ & 23.8 & 71.3 & 0.000 & 261.64 & 0.045 & Dry \\
\hline HiVol 10* & $\begin{array}{l}7 / 31 / 2011 \\
20: 06\end{array}$ & $\begin{array}{l}7 / 31 / 2011 \\
23: 57\end{array}$ & 16.4 & 72.4 & 0.000 & 262.85 & 0.055 & Dry \\
\hline HiVol 11 & $\begin{array}{l}8 / 1 / 2011 \\
0: 01\end{array}$ & $\begin{array}{l}8 / 1 / 2011 \\
4: 01\end{array}$ & 14.0 & 47.0 & 0.000 & 264.14 & 0.064 & Dry \\
\hline HiVol 12* & $\begin{array}{l}8 / 1 / 2011 \\
4: 03\end{array}$ & $\begin{array}{l}8 / 1 / 2011 \\
8: 03\end{array}$ & 16.8 & 24.1 & 0.000 & 264.60 & 0.061 & Dry \\
\hline HiVol 13 & $\begin{array}{l}8 / 1 / 2011 \\
8: 06\end{array}$ & $\begin{array}{l}8 / 1 / 2011 \\
20: 00\end{array}$ & 22.4 & 40.2 & 0.002 & 272.02 & 0.065 & Dry \\
\hline HiVol 14* & $\begin{array}{l}8 / 1 / 2011 \\
20: 04\end{array}$ & $\begin{array}{l}8 / 2 / 2011 \\
20: 26\end{array}$ & 14.5 & 59.3 & 0.090 & 323.79 & 0.163 & Rainy \\
\hline HiVol 15* & $\begin{array}{l}8 / 2 / 2011 \\
20: 28\end{array}$ & $\begin{array}{l}8 / 3 / 2011 \\
20: 04\end{array}$ & 15.2 & 83.4 & 0.019 & 319.09 & 0.241 & Rainy \\
\hline HiVol 16 & $\begin{array}{l}8 / 3 / 2011 \\
20: 06\end{array}$ & $\begin{array}{l}8 / 4 / 2011 \\
0: 07\end{array}$ & 12.1 & 93.1 & 0.023 & 311.64 & 0.281 & Rainy \\
\hline HiVol 17* & $\begin{array}{l}8 / 4 / 2011 \\
0: 09\end{array}$ & $\begin{array}{l}8 / 4 / 2011 \\
4: 10\end{array}$ & 10.1 & 81.0 & 0.000 & 345.84 & 0.348 & Rainy \\
\hline HiVol 18* & $\begin{array}{l}8 / 4 / 2011 \\
4: 13\end{array}$ & $\begin{array}{l}8 / 4 / 2011 \\
8: 12\end{array}$ & 12.3 & 45.2 & 0.000 & 316.78 & 0.290 & Rainy \\
\hline HiVol 19* & $\begin{array}{l}8 / 4 / 2011 \\
8: 15\end{array}$ & $\begin{array}{l}8 / 4 / 2011 \\
12: 17\end{array}$ & 20.6 & 66.2 & 0.000 & 268.53 & 0.131 & Rainy \\
\hline
\end{tabular}




\begin{tabular}{|c|c|c|c|c|c|c|c|c|}
\hline HiVol 20* & $\begin{array}{l}8 / 4 / 2011 \\
12: 19\end{array}$ & $\begin{array}{l}8 / 4 / 2011 \\
15: 57\end{array}$ & 16.5 & 89.9 & 1.088 & 345.72 & 0.114 & $\begin{array}{l}\text { Other }^{\dagger} \\
\text { (Rainy) }\end{array}$ \\
\hline HiVol 21* & $\begin{array}{l}8 / 4 / 2011 \\
16: 00\end{array}$ & $\begin{array}{l}8 / 4 / 2011 \\
20: 12\end{array}$ & 12.3 & 91.5 & 0.021 & 340.62 & 0.189 & $\begin{array}{l}\text { Other }^{\dagger} \\
\text { (Rainy) }\end{array}$ \\
\hline HiVol 22 & $\begin{array}{l}8 / 4 / 2011 \\
20: 14\end{array}$ & $\begin{array}{l}8 / 6 / 2011 \\
20: 03\end{array}$ & 16.3 & 66.8 & 0.001 & 303.36 & 0.170 & Rainy \\
\hline HiVol 23 & $\begin{array}{l}8 / 6 / 2011 \\
20: 05\end{array}$ & $\begin{array}{l}8 / 7 / 2011 \\
20: 05\end{array}$ & 19.3 & 46.2 & 0.000 & 280.55 & 0.097 & $\begin{array}{l}\text { Dry }^{\dagger} \\
\text { (Rainy) }\end{array}$ \\
\hline HiVol 24 & $\begin{array}{l}8 / 7 / 2011 \\
20: 12\end{array}$ & $\begin{array}{l}8 / 8 / 2011 \\
19: 48\end{array}$ & 16.4 & 36.0 & 0.000 & 261.57 & 0.072 & Dry \\
\hline HiVol 25 & $\begin{array}{l}8 / 8 / 2011 \\
19: 49\end{array}$ & $\begin{array}{l}8 / 9 / 2011 \\
20: 11\end{array}$ & 18.6 & 39.6 & 0.000 & 276.79 & 0.082 & Other \\
\hline HiVol 27 & $\begin{array}{l}8 / 9 / 2011 \\
20: 13\end{array}$ & $\begin{array}{l}8 / 10 / 2011 \\
20: 02\end{array}$ & 15.7 & 41.5 & 0.000 & 273.60 & 0.089 & Other \\
\hline HiVol 28 & $\begin{array}{l}8 / 10 / 2011 \\
20: 05\end{array}$ & $\begin{array}{l}8 / 11 / 2011 \\
19: 53\end{array}$ & 17.0 & 61.3 & 0.001 & 300.35 & 0.061 & $\begin{array}{l}\text { Other }^{\dagger} \\
\text { (Rainy) }\end{array}$ \\
\hline HiVol 29 & $\begin{array}{l}8 / 11 / 2011 \\
19: 54\end{array}$ & $\begin{array}{l}8 / 12 / 2011 \\
19: 51\end{array}$ & 16.4 & 51.3 & 0.000 & 267.80 & 0.061 & Dry \\
\hline HiVol 30 & $\begin{array}{l}8 / 12 / 2011 \\
19: 52\end{array}$ & $\begin{array}{l}8 / 13 / 2011 \\
19: 47\end{array}$ & 17.3 & 52.2 & 0.000 & 291.40 & 0.075 & Dry \\
\hline HiVol 31 & $\begin{array}{l}8 / 13 / 2011 \\
19: 48\end{array}$ & $\begin{array}{l}8 / 14 / 2011 \\
19: 54\end{array}$ & 18.5 & 53.3 & 0.000 & 264.41 & 0.082 & Dry \\
\hline HiVol 32 & $\begin{array}{l}8 / 14 / 2011 \\
19: 55\end{array}$ & $\begin{array}{l}8 / 15 / 2011 \\
20: 05\end{array}$ & 17.5 & 57.8 & 0.000 & 281.19 & 0.073 & Dry \\
\hline HiVol 33* & $\begin{array}{l}8 / 15 / 2011 \\
20: 06\end{array}$ & $\begin{array}{l}8 / 16 / 2011 \\
19: 47\end{array}$ & 15.0 & 51.2 & 0.003 & 278.96 & 0.080 & Dry \\
\hline HiVol 35* & $\begin{array}{l}8 / 16 / 2011 \\
19: 48\end{array}$ & $\begin{array}{l}8 / 17 / 2011 \\
20: 05\end{array}$ & 16.9 & 63.0 & 0.000 & 303.81 & 0.101 & Dry \\
\hline HiVol 36* & $\begin{array}{l}8 / 17 / 2011 \\
20: 06\end{array}$ & $\begin{array}{l}8 / 18 / 2011 \\
19: 47\end{array}$ & 18.2 & 55.7 & 0.000 & 295.59 & 0.072 & Dry \\
\hline HiVol 37* & $\begin{array}{l}8 / 18 / 2011 \\
19: 48\end{array}$ & $\begin{array}{l}\text { 8/19/2011 } \\
20: 07\end{array}$ & 17.8 & 41.8 & 0.000 & 262.09 & 0.074 & Dry \\
\hline HiVol 38* & $\begin{array}{l}8 / 19 / 2011 \\
20: 08\end{array}$ & $\begin{array}{l}8 / 20 / 2011 \\
20: 08\end{array}$ & 18.1 & 47.3 & 0.000 & 265.92 & 0.071 & $\begin{array}{l}\text { Other }^{\dagger} \\
\text { (Dry) }\end{array}$ \\
\hline
\end{tabular}

Table S1: Summary information for each hi-volume filter sample including: start and stop times (local time; U.S. date format $\mathrm{m} / \mathrm{dd} / \mathrm{yyyy}$ ), average air temperature, relative humidity, rain amount (normalized to 2.0) leaf wetness, number ratio of fluorescent particles from the UV-APS, and wetness category determined as described in Section 3.1. Star symbol (*, first column) indicates samples used in fungal DNA determination. Cross symbol ( ${ }^{\dagger}$, last column) indicates that category assignment was manually changed from the algorithm determination (original category in parentheses). $\mathrm{N}_{\mathrm{f}}$ represents the number of fluorescent particles, $\mathrm{N}_{\text {tot }}$ represents the number of total particles as measured by the UV-APS. 


\begin{tabular}{|l|l|l|}
\hline \multicolumn{1}{|c|}{ Figure } & \multicolumn{2}{c|}{ Linear Fit Parameters } \\
\hline & \multicolumn{1}{|c|}{ Rainy } & \multicolumn{1}{c|}{ Dry } \\
\hline $5 . c$ & $y=38.0 x-21.8$ & $y=2.0 x+8.1$ \\
\hline $5 . d$ & $y=54.9 x-37.5$ & $y=2.9 x+8.3$ \\
\hline $5 . e$ & $y=32.0 x+11.9$ & $y=18.8 x+6.9$ \\
\hline $5 . f$ & $y=41.6 x+14.6$ & $y=9.9 x+9.2$ \\
\hline
\end{tabular}

25

26 Table S2: Linear equation fit parameters for Rainy and Dry conditions for Figure 5c-f. Each equation

27 represents the linear trend line for correlations of arabitol $(5 \mathrm{c}, \mathrm{e})$ or mannitol $(5 \mathrm{~d}, \mathrm{f})$ with UV-APS FAP

28 mass $(5 \mathrm{c}, \mathrm{d})$ or WIBS Cl 1 FAP mass $(5 \mathrm{e}, \mathrm{f})$. 


\begin{tabular}{|l|l|l|}
\hline \multicolumn{1}{|c|}{ Figure } & \multicolumn{2}{c|}{ Linear Fit Parameters } \\
\hline & \multicolumn{1}{|c|}{ Rainy } & \multicolumn{1}{c|}{ Dry } \\
\hline $6 . d$ & $y=0.4 x+11646$ & $y=0.2 x+5064$ \\
\hline $6 . e$ & $y=0.3 x+9613$ & $y=0.2 x+1939$ \\
\hline $6 . f$ & $y=0.004 x+236$ & $y=0.01 x+83$ \\
\hline $6 . g$ & $y=0.9 x+15514$ & $y=1.4 x+5389$ \\
\hline $6 . h$ & $y=0.9 x+12683$ & $y=1.1 x+4094$ \\
\hline $6 . i$ & $y=0.005 x+313$ & $y=0.05 x+190$ \\
\hline
\end{tabular}

Table S3: Linear equation fit parameters for Rainy and Dry conditions for Figure 6d-i. Each equation represents the linear trend line for correlations of estimated fungal spores $\left(\mathrm{m}^{-3}\right)$ from $(6 \mathrm{~d}, \mathrm{~g})$ arabitol, $(6 \mathrm{e}, \mathrm{h})$ mannitol or $(6 \mathrm{f}, \mathrm{i})$ colony forming units $(\mathrm{CFU})$ with $(6 \mathrm{~d}, \mathrm{e}, \mathrm{f}) \mathrm{UV}-\mathrm{APS}$ FAPs or $(6 \mathrm{~g}, \mathrm{~h}, \mathrm{i})$ WIBS Cl 1 FAPs. 


\begin{tabular}{|l|l|l|l|}
\hline \multicolumn{5}{|c|}{ Particle Mass Percentage (\%) } \\
\hline & Dry & Rainy & Other \\
\hline C11 & $2.15 \pm 1.38$ & $16.98 \pm 10.14$ & $4.03 \pm 3.42$ \\
\hline C12 & $4.72 \pm 1.43$ & $6.01 \pm 1.57$ & $6.68 \pm 2.38$ \\
\hline Cl3 & $19.92 \pm 5.81$ & $13.22 \pm 5.78$ & $23.79 \pm 10.60$ \\
\hline C14 & $4.44 \pm 1.64$ & $8.83 \pm 3.73$ & $6.53 \pm 3.45$ \\
\hline FL 1 & $8.42 \pm 3.37$ & $62.05 \pm 35.10$ & $24.70 \pm 23.61$ \\
\hline FL 2 & $18.51 \pm 4.02$ & $71.55 \pm 31.34$ & $38.26 \pm 24.77$ \\
\hline FL 3 & $36.79 \pm 6.26$ & $85.95 \pm 28.23$ & $61.77 \pm 28.29$ \\
\hline FL & $38.01 \pm 6.34$ & $87.99 \pm 28.53$ & $64.92 \pm 30.66$ \\
\hline UV-APS FAP & $25.53 \pm 2.99$ & $51.50 \pm 14.83$ & $32.87 \pm 9.45$ \\
\hline & \multicolumn{2}{|c|}{ Total Particle Mass $\left(\mu \mathrm{g} \mathrm{m}{ }^{-3}\right)$} \\
\hline UV-APS Total & $3.70 \pm 1.11$ & $2.70 \pm 0.58$ & $4.85 \pm 2.56$ \\
\hline
\end{tabular}

Table S4: Percentage of particle mass in various UV-LIF instrument categories and total particle mass. Each mass value compared to total particle mass, determined using UV-APS number size distributions, converted to a mass for particles of aerodynamic diameter $0.5-10 \mu \mathrm{m}$ and using particle mass density of $1.5 \mathrm{~g} \mathrm{~cm}^{-3}$. WIBS particles were integrated into total number over the same size range in optical diameter and using unity density. Ranges shown are standard deviation of 5-minute time averages. 


\begin{tabular}{|c|c|c|c|}
\hline Particle Type & FL1 Fluorescence Intensity & FL2 Fluorescence Intensity & FL3 Fluorescence Intensity \\
\hline A & I Threshold & & \\
\hline B & & | Threshold & \\
\hline C & & & | Threshold \\
\hline AB & I Threshold & | Threshold & \\
\hline AC & | Threshold & & | Threshold \\
\hline BC & & | Threshold & | Threshold \\
\hline ABC & 1 Threshold & | Threshold & | Threshold \\
\hline
\end{tabular}

Figure S1: Particle type assignment strategy for WIBS data. Particle category type defined as fluorescent 43 in a given channel when the fluorescence intensity (I) in channel FL1, FL2, or FL3 is greater than the 44 threshold value, defined as blank + 3SD. Colors correspond to particle type used also in Figures 2-3. 

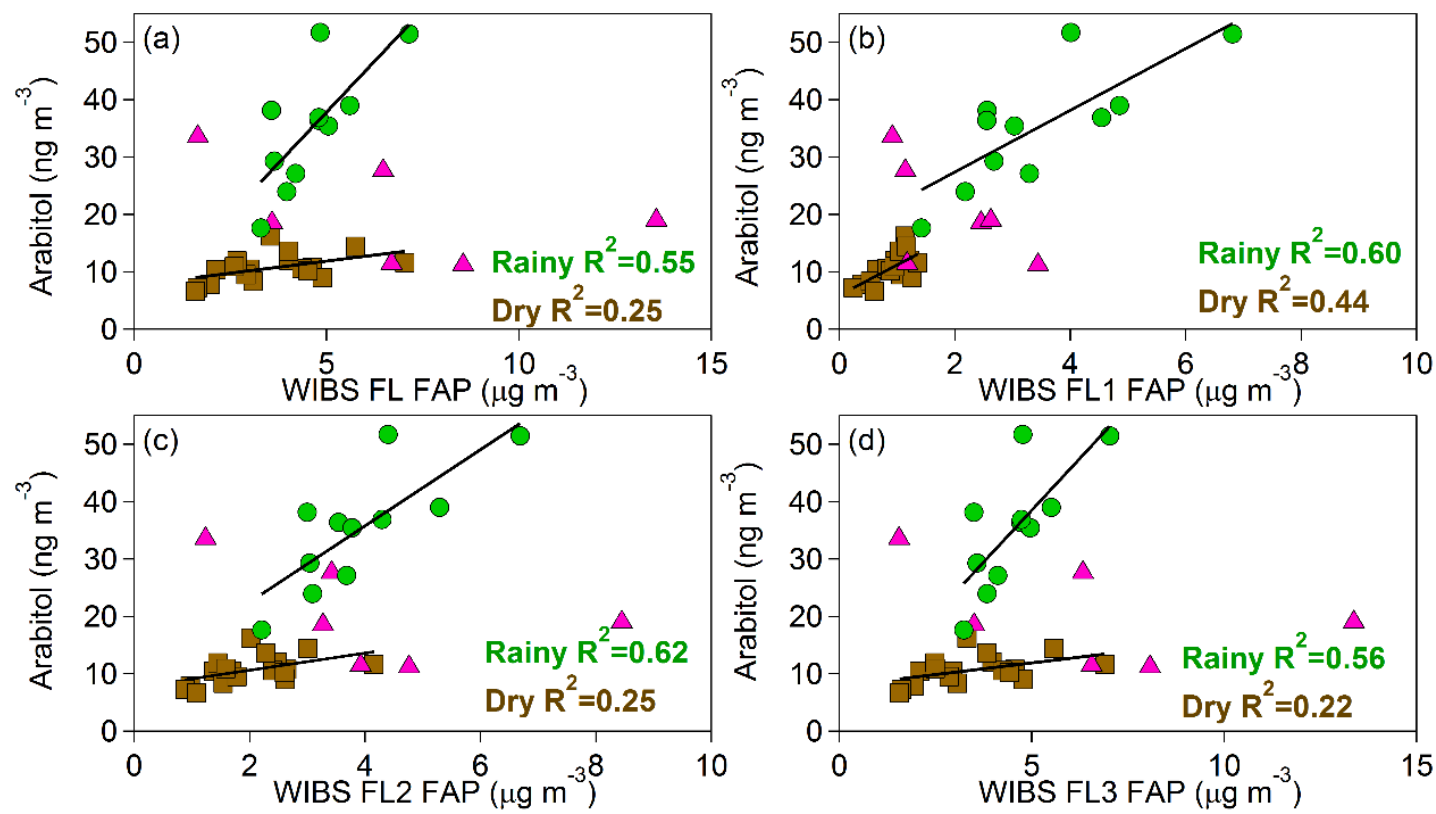

Figure S2: Atmospheric arabitol concentration $\left(\mathrm{ng} \mathrm{m}^{-3}\right)$ correlated with WIBS fluorescent particle mass $\left(\mu \mathrm{g} \mathrm{m}^{-3}\right)$ (a) any fluorescent particle, FL; (b) particles fluorescent in channel 1, FL1; (c) particles fluorescent in channel 2, FL2; (d) particles fluorescent in channel 3, FL3. R ${ }^{2}$ value shown for each fit in a-d. Linear fit parameter are shown in the table below.

\begin{tabular}{|l|l|l|}
\hline \multicolumn{1}{|c|}{ Figure } & \multicolumn{2}{c|}{ Linear Fit Parameters } \\
\hline & \multicolumn{1}{|c|}{ Rainy } & \multicolumn{1}{c|}{ Dry } \\
\hline S2.a & $y=7.1 \mathrm{x}+2.4$ & $\mathrm{y}=0.8 \mathrm{x}+7.6$ \\
\hline S2.b & $\mathrm{y}=5.4 \mathrm{x}+16.7$ & $\mathrm{y}=5.3 \mathrm{x}+5.9$ \\
\hline S2.c & $\mathrm{y}=6.6 \mathrm{x}+9.2$ & $\mathrm{y}=1.5 \mathrm{x}+7.6$ \\
\hline S2.d & $\mathrm{y}=7.2 \mathrm{x}+2.4$ & $\mathrm{y}=0.81 \mathrm{x}+7.8$ \\
\hline
\end{tabular}

52 Linear equation fit parameters for Rainy and Dry conditions for Figure S2a-d. Each equation represents 53 the linear trend line for correlations of arabitol $\left(\mathrm{ng} \mathrm{m}^{-3}\right)$ with WIBS fluorescent channel particle mass $(\mu \mathrm{g}$ $54 \mathrm{~m}^{-3}$ ). (a) any fluorescent particle, FL; (b) particles fluorescent in channel 1, FL1; (c) particles fluorescent 55 in channel 2, FL2; (d) particles fluorescent in channel 3, FL3. 

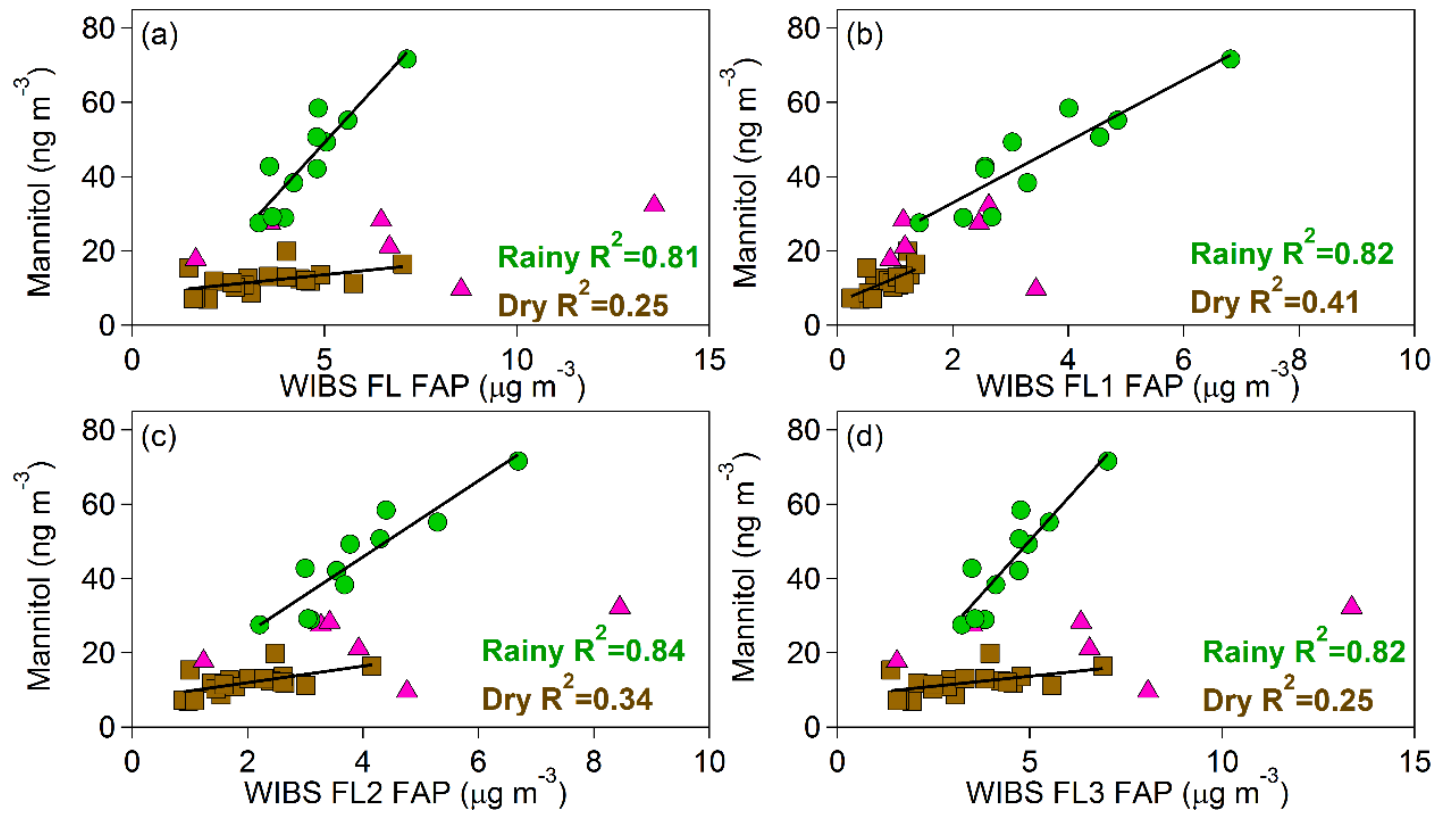

Figure S3: Atmospheric mannitol concentration $\left(\mathrm{ng} \mathrm{m}^{-3}\right)$ correlated with WIBS fluorescent particle mass $\left(\mu \mathrm{g} \mathrm{m}^{-3}\right)$ (a) any fluorescent particle, FL; (b) particles fluorescent in channel 1, FL1; (c) particles fluorescent in channel 2, FL2; (d) particles fluorescent in channel 3, FL3. $\mathrm{R}^{2}$ value shown for each fit in ad. Linear fit parameter are shown in the table below.

\begin{tabular}{|l|l|l|}
\hline \multicolumn{1}{|c|}{ Figure } & \multicolumn{2}{c|}{ Linear Fit Parameters } \\
\hline & \multicolumn{1}{|c|}{ Rainy } & \\
\hline S3.a & $y=11.3 x-7.5$ & $y=1.1 x+8.2$ \\
\hline S3.b & $y=8.3 x+16.4$ & $y=6.5 x+6.2$ \\
\hline S3.c & $y=10.3 x+4.9$ & $y=2.2 x+7.5$ \\
\hline S3.d & $y=11.5 x-7.4$ & $y=1.1 x+8.2$ \\
\hline
\end{tabular}

Linear equation fit parameters for Rainy and Dry conditions for Figure S3a-d. Each equation represents 

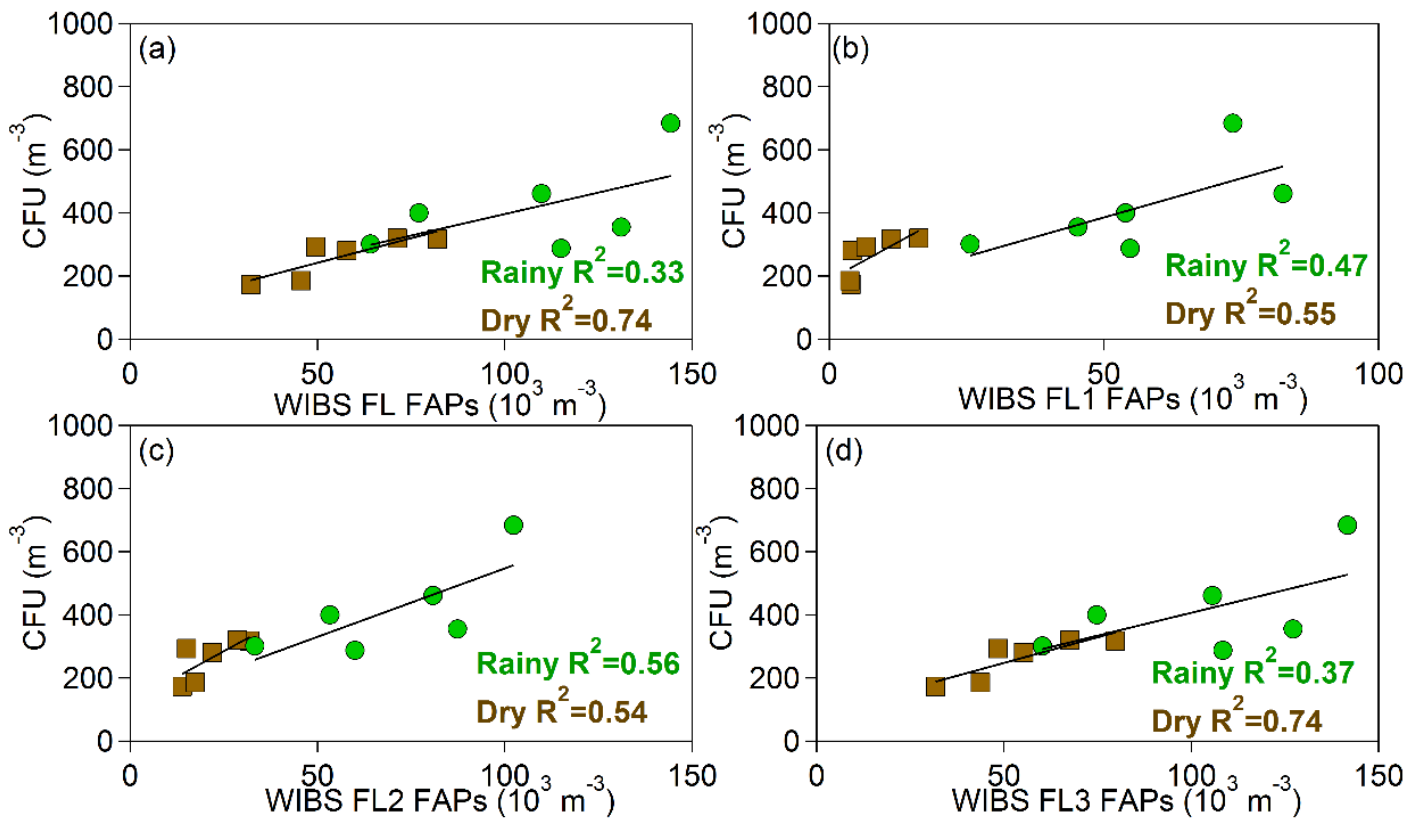

Figure S4: Atmospheric colony forming unit (CFU) concentration $\left(\mathrm{CFU} \mathrm{m}{ }^{-3}\right)$ correlated with WIBS fluorescent particle $\left(\mathrm{m}^{-3}\right)$ (a) any fluorescent particle, FL; (b) particles fluorescent in channel 1, FL1; (c) particles fluorescent in channel 2, FL2; (d) particles fluorescent in channel 3, FL3. R ${ }^{2}$ value shown for each fit in a-d. Linear fit parameter are shown in the table below.

72

\begin{tabular}{|l|l|l|}
\hline Figure & Rainy Linear Parameters & Dry Linear Parameters \\
\hline S4.a & $\mathrm{y}=0.003 \mathrm{x}+124$ & $\mathrm{y}=0.003 \mathrm{x}+86$ \\
\hline S4.b & $\mathrm{y}=0.005 \mathrm{x}+138$ & $\mathrm{y}=0.009 \mathrm{x}+189$ \\
\hline S4.c & $\mathrm{y}=0.004 \mathrm{x}+113$ & $\mathrm{y}=0.006 \mathrm{x}+122$ \\
\hline S4.d & $\mathrm{y}=0.003 \mathrm{x}+118$ & $\mathrm{y}=0.003 \mathrm{x}+84$ \\
\hline
\end{tabular}

73

74 Linear equation parameters for Rainy and Dry conditions for Figure S4a-d. Each equation represents the

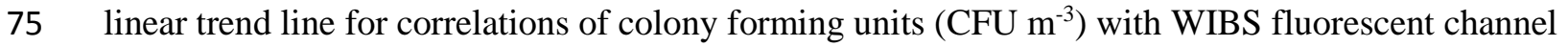

76 particles $\left(\mathrm{m}^{-3}\right)$. (a) any fluorescent particle, FL; (b) particles fluorescent in channel 1, FL1; (c) particles

77 fluorescent in channel 2, FL2; (d) particles fluorescent in channel 3, FL3. 